\title{
On Spectrum Sensing for TV White Space in China
}

\author{
Christian Kocks, ${ }^{1}$ Alexander Viessmann, ${ }^{1}$ Peter Jung, ${ }^{1}$ Lei Chen, ${ }^{2}$ \\ Qiu Jing, ${ }^{2}$ and Rose Qingyang $\mathrm{Hu}^{3}$ \\ ${ }^{1}$ Department of Communication Technologies, University of Duisburg-Essen, 47057 Duisburg, Germany \\ ${ }^{2}$ Communication Technology Research Department, Huawei Tech. Co., Ltd, Chengdu, Sichuan Province 610041, China \\ ${ }^{3}$ Department of Electrical and Computer Engineering, Utah State University, Logan, UT 84322, USA
}

Correspondence should be addressed to Christian Kocks, christian.kocks@kt.uni-due.de

Received 13 February 2012; Accepted 7 May 2012

Academic Editor: Luca Ronga

Copyright ( $\odot 2012$ Christian Kocks et al. This is an open access article distributed under the Creative Commons Attribution License, which permits unrestricted use, distribution, and reproduction in any medium, provided the original work is properly cited.

In the field of wireless communications the idea of cognitive radio is of utmost interest. Due to its advantageous propagation properties, the TV white space can be considered to become the first commercial application of cognitive radio. It allows the usage of secondary communication systems at non-occupied frequency bands. Within this paper, spectrum sensing algorithms are introduced for the three predominant Chinese TV standards DTMB, CMMB, and PAL-D/K. A prototype platform is presented and its underlying architecture based on a combination of DSP and FPGA is illustrated including the setup of the cognitive radio application. Furthermore, the performance of the sensing algorithms implemented on the prototype platform is shown in comparison to simulation results.

\section{Introduction}

The recent decade revealed increasing interests in the field of cognitive radio (CR) for wireless communication systems. It is considered as a key technology for significantly alleviating spectrum scarcity.

The TV white space (TVWS), which refers to nonoccupied frequency bands in the TV spectrum, that is below $900 \mathrm{MHz}$, is a desirable target for CR-based spectrum sharing due to its advantageous propagation properties compared to other frequency ranges on the one hand and due to its low utilization ratio on the other hand [1]. Hence, deploying CR mechanisms in TVWS will probably become the first commercial application that brings CR from concept to reality. In the United States, the FCC has already made an official request to allow unlicensed users reusing TV bands without causing interference to incumbent users [2]. In other countries, the corresponding regulatory authorities such as the CEPT in Europe are developing regulations on the unlicensed usage in TVWS as well. Besides the regulatory authorities, working groups such as IEEE 802.22 [3] have started the standardization for cognitive radio applications.
Spectrum sensing is a key element of CR and its application to TVWS has been widely studied. However, a variety of different TV standards exists which may differ from country to country, especially for digital TV standards. While in North America ATSC (Advanced Television Systems Committee) is deployed, in Europe, South Asia, and Africa, DVB-T/H (Digital Video BroadcastingTerrestrial/Handheld) plays the predominant role. Further standards such as ISDB (Integrated Services Digital Broadcasting) developed in Japan or DMB (Digital Multimedia Broadcasting) developed in Korea are also used in various countries [4]. As a result, it is hardly feasible to design a universal sensing algorithm for all TV standards. This paper focuses on spectrum sensing for Chinese TV standards using an autocorrelation approach. Although the autocorrelation approach has been widely studied for the purpose of spectrum sensing, the application to Chinese TV standards has not been considered thoroughly yet.

In China, mainly three terrestrial and handheld TV standards are deployed: DTMB (Digital Terrestrial Multimedia Broadcast) [5] for terrestrial reception, CMMB (China Mobile Multimedia Broadcasting) [6] for handheld reception 
and PAL-D/K (Phase Alternating Line) [7] for analog TV. While other countries such as the USA have already stopped the provision of analog TV, the nationwide switchover from analog to digital TV will not occur until the year 2015 . Therefore, the analog TV will still coexist with the digital TV for many years to come. However, measurements on the channel occupancy rate revealed many unused channels although the analog TV is still provided. As a result, the detection of both analog and digital signals is necessary for CR implementations.

The spectrum sensing requirements in China are based on the IEEE 802.22 specifications. That is, the sensing threshold is $-114 \mathrm{dBm}$ for $6 \mathrm{MHz}$ channel bandwidth yielding $-112.8 \mathrm{dBm}$ for $8 \mathrm{MHz}$ wide channels. For analog TV, the threshold is $-114 \mathrm{dBm}$ in $100 \mathrm{kHz}$ bandwidth yielding $-95.0 \mathrm{dBm}$ in $8 \mathrm{MHz}$ bandwidth.

The United Kingdom and the United States are the most active countries in exploiting the unlicensed usage of TVWS. The spectrum sensing technology for ATSC signals has been intensively studied. Several detection algorithms for ATSC and its analog predecessor NTSC (National Television System Committee) can be found in the IEEE standard 802.22 [8]. In 2008, a sensing prototype test campaign was organized by the FCC [9]. As an example, Motorola, Philips, and I2R have submitted their prototype designs which have been tested both in the laboratory as well as in the field. The results showed that the ATSC and NTSC signals can be detected correctly with a certain probability. As another widely used TV standard, DVB-T has also been intensively studied with respect to spectrum sensing. In [10], a robust sensing approach is discussed using a prototype sensor developed by Philips. Several detection algorithms for a Chinese standard, that is DTMB, have also been studied and published $[11,12]$. In this paper, an experimental spectrum sensing prototype platform for Chinese TV standards is presented and results are discussed.

This paper is structured as follows. Section 2 gives an introduction to the Chinese TV standards DTMB, CMMB, and PAL-D/K. The corresponding sensing algorithms are presented in Section 3. In Section 4, the prototype platform is illustrated including the signal flow for the spectrum sensing operation. Section 5 shows selected results in a comparison between the simulated algorithms' performance and the performance measured with the prototype platform. Finally, a conclusion is given.

\section{Chinese TV Standards}

The intention of this section is to give a brief overview of the various Chinese TV standards. The focus is on the main aspects which are relevant for feature-based signal detection. For a full description of the TV systems, please refer to [5-7].

2.1. DTMB. DTMB, also referred to as DMB-T (Digital Multimedia Broadcast-Terrestrial), is a mandatory TV standard in China. DTMB can be used in either single-carrier or in multi-carrier mode. Three FEC (Forward Error Correction) code rates, five modulation orders, and two interleaving depths are specified for DTMB [5]. A block diagram of a DTMB transmitter is shown in Figure 1.

DTMB defines three different header types with different lengths. The frame body itself has a fixed length of $500 \mu \mathrm{s}$. The frame structure of DTMB including the different header types is illustrated in Figure 2. The frames are hierarchically structured in a calendar day frame, a minute frame, and a super frame. One calendar day frame lasts for 24 hours and contains 1440 minute frames with a duration of one minute each. Each minute frame, in turn, consists of 480 super frames. One super frame consists of either 225 frames with frame header mode 1 or of 216 frames with frame header mode 2 or of 200 frames with frame header mode 3.

The three frame headers are generated by different generator polynomials which are [5]

$$
G_{1}(x)=1+x+x^{5}+x^{6}+x^{8}
$$

for mode 1 ,

$$
G_{2}(x)=1+x^{3}+x^{10}
$$

for mode 2, and

$$
G_{3}(x)=1+x^{2}+x^{7}+x^{8}+x^{9}
$$

for mode 3. The generation of the sequence can be realized by a linear feedback shift register. The selection on the frame header mode depends on the desired coverage and is fixed once the transmission has begun.

2.2. $C M M B$. CMMB is a system fully based on the wellknown OFDM (Orthogonal Frequency Division Multiplexing). The transmitter structure is depicted in Figure 3. A combination of Reed-Solomon (RS) and Low-Density Parity-Check (LDPC) codes is used for FEC. Unlike many other OFDM systems such as DVB-T [13], the OFDM symbol of length $T_{0}$ in time-domain is not only extended by inserting a cyclic prefix (length $T_{1}$ ), but it is also extended by a pre-guard interval and a post-guard interval of length $T_{\mathrm{GI}}$ each. As illustrated in Figure 4, the post-guard interval of a certain OFDM symbol in CMMB overlaps with the preguard interval of the subsequent symbol [6].

In CMMB, one frame has a duration of $1 \mathrm{~s}$ and consists of 40 time slots. Each time slot contains one beacon and 53 OFDM symbols. The beacon contains a transmitter identification field and two synchronization signals. The OFDM symbols consist of data-bearing subcarriers as well as of pilot subcarriers. These pilot subcarriers are subdivided into continual pilots and scattered pilots [6].

2.3. $P A L-D / K$. A variety of different PAL-based standards exist which mainly differ in the channel bandwidth or in the underlying modulation scheme. The PAL standard used in China is called PAL-D/K with $8 \mathrm{MHz}$ channel bandwidth, $50 \mathrm{~Hz}$ field frequency, and 625 lines per frame [7]. A PAL signal consists of separate video and audio parts. Within this paper, only the bandwidth occupied by the video part is subject to spectrum sensing. The video signal used in PAL 


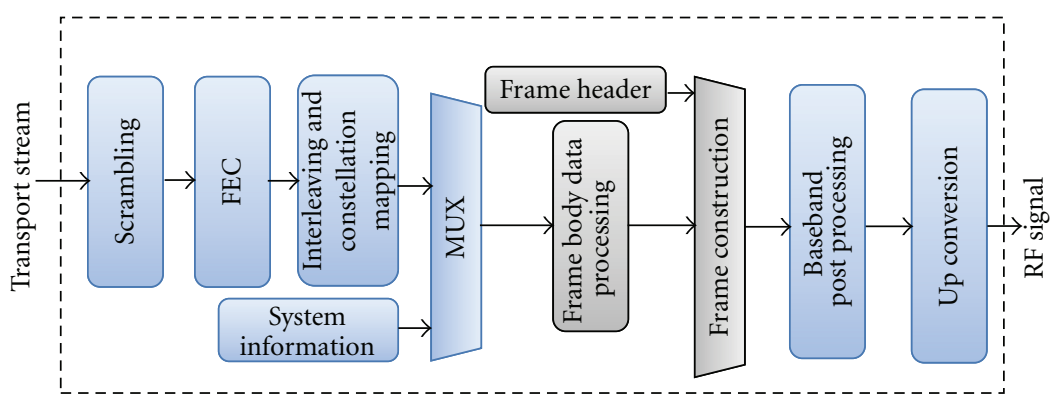

FIgURE 1: DTMB transmitter [5].

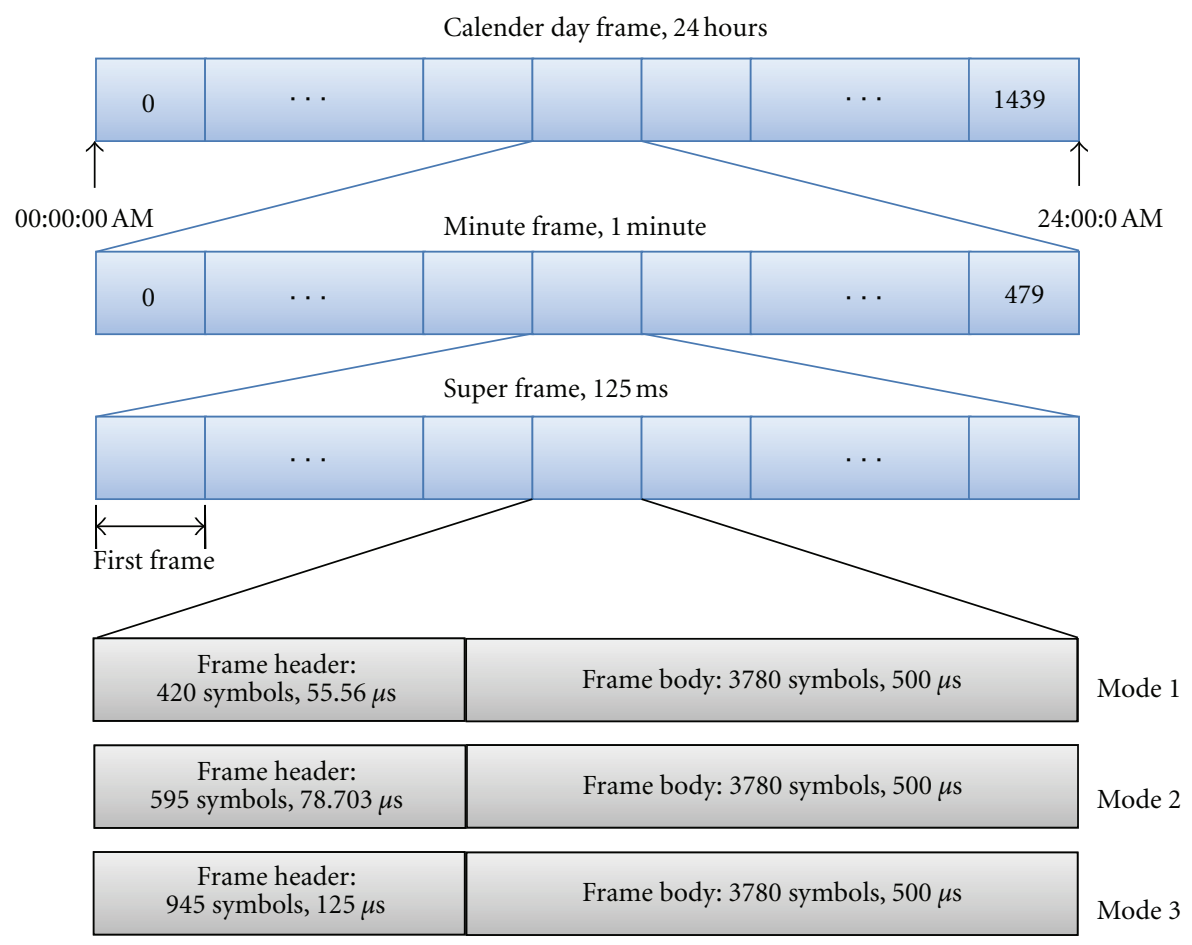

FIGURE 2: DTMB frame structure [5].

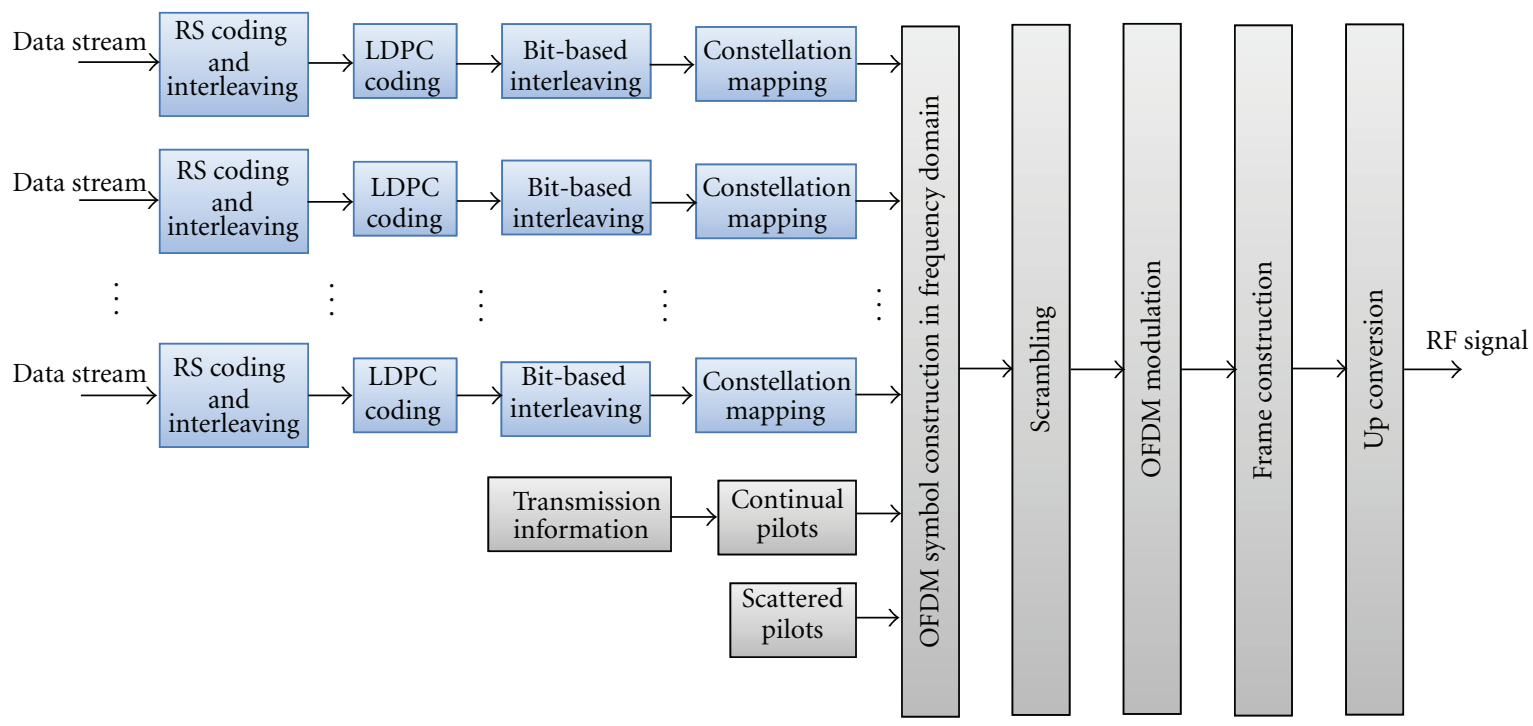

Figure 3: CMMB transmitter [6]. 


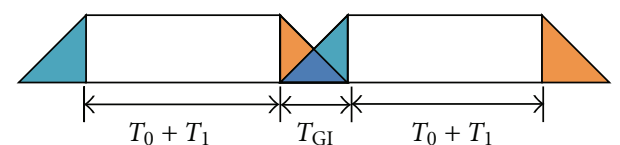

FIgURE 4: CMMB symbol overlapping.

is a CVBS (Color Video, Blanking and Sync) signal which is an extension to the monochrome VBS (Video, Blanking and Sync) signal. A snapshot of a VBS signal is depicted in Figure 5. In addition to the video signal itself, the VBS signal has some additional components which are required for example for synchronization at the receiver. The blacklevel signal components after and before the video signal are referred to as front porch and back porch, respectively. The time values shown in Figure 5 are compliant to the PAL-D/K standard. The total duration of one line is $64 \mu$ s resulting in a line frequency of $15625 \mathrm{~Hz}$ [7].

\section{Spectrum Sensing Algorithms}

After the brief introduction to the various Chinese TV standards, this section describes the spectrum sensing algorithms. All algorithms have in common that they are based on autocorrelation of the digital baseband signal. In general, the autocorrelation function $\varphi_{s s}(t)$ of a complex signal $s(t)$ is defined as

$$
\varphi_{s s}(\tau)=\int_{-\infty}^{\infty} s^{*}(t) s(t+\tau) d t
$$

where $(\cdot)^{*}$ denotes the complex conjugation.

3.1. DTMB. In DTMB, the frame header appears periodically at the beginning of each frame which can be exploited for the sensing operation. The presented autocorrelationbased sensing algorithm for DTMB can be divided into three stages:

(i) autocorrelation stage,

(ii) comb-correlation stage,

(iii) decision stage.

A flow diagram of the algorithm is shown in Figure 6. For the autocorrelation the digitized baseband signal is multiplied with a delayed and complex conjugated version of the signal where the delay itself depends on the frame header mode. In case the frame header mode is unknown, the algorithm needs to be carried out for each frame header mode separately. The running average filter cumulates a certain number of the multiplication output samples. Resulting from the periodical appearance of the frame header, the first stage's output is applied to a comb correlator which is a correlation with a Dirac comb $g(t)$ with a distance $\Delta t$ corresponding to the frame header period, that is,

$$
g(t)=\sum_{k=-\infty}^{\infty} \delta(t-k \cdot \Delta t) .
$$

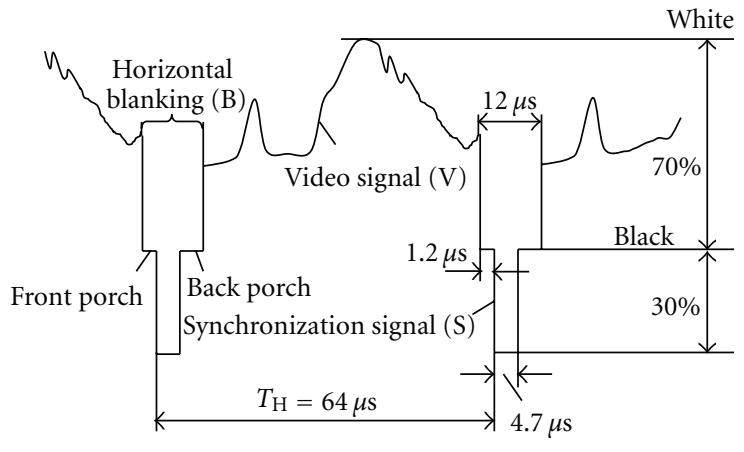

FIGURE 5: VBS signal.

This stage allows collecting the energy of all frames within the sensing period. The squared magnitude of the cumulated comb-correlation output $\varphi_{c c}$ is given to the decision stage. In this stage, the ratio $\lambda$ of the maximum and the average of the previous stage's output is calculated:

$$
\lambda=\frac{\max \left(\varphi_{\mathrm{cc}}(t)\right)}{\overline{\varphi_{\mathrm{cc}}(t)}} .
$$

By applying a soft-decision metric to $\lambda$, a measure for the probability of the presence of a DTMB signal is generated. Furthermore, comparing the ratio with a threshold $\Gamma$ gives a hard-decision on the presence of a DTMB signal. This threshold is generated by using a threshold metric based on the available sensing interval $t_{\text {sense }}$ and the desired false-alarm probability $P_{\text {fa }}$.

By using the ratio $\lambda$ for making the decision about the presence of a DTMB signal, the presented algorithm is robust against dynamic range variations as well as varying signal-tonoise ratios and, thus, independent of the underlying AGC (Automatic Gain Control) implementation.

3.2. $C M M B$. The sensing algorithm for $\mathrm{CMMB}$ is very similar to the sensing algorithm for DTMB. As shown in Section 2, CMMB uses a cyclic repetition of certain parts of the OFDM symbol, denoted as cyclic prefix. Since this cyclic prefix equals the last part of the corresponding OFDM symbol, it is well suited for the sensing operation. The general data flow of the algorithm is identical to the DTMB algorithm depicted in Figure 6. However, the timings must be adapted according to the CMMB parameters.

3.3. $P A L-D / K$. The sensing algorithm for PAL-D/K relies on the periodicity of certain parts of the CVBS signal as depicted in Figure 5. The CVBS signal exhibits a periodic pattern of the synchronization pulses in every transmitted line of the resulting TV picture. In addition to the synchronization pulses itself with a length of $t_{\text {hsync }}=4.7 \mu \mathrm{s}$, the front as well as the back porch with lengths of $t_{\mathrm{fp}}=1.2 \mu \mathrm{s}$ and $t_{\mathrm{bp}}=6.1 \mu \mathrm{s}$, respectively, can be used for sensing purposes. The time between two consecutive synchronization pulses is 


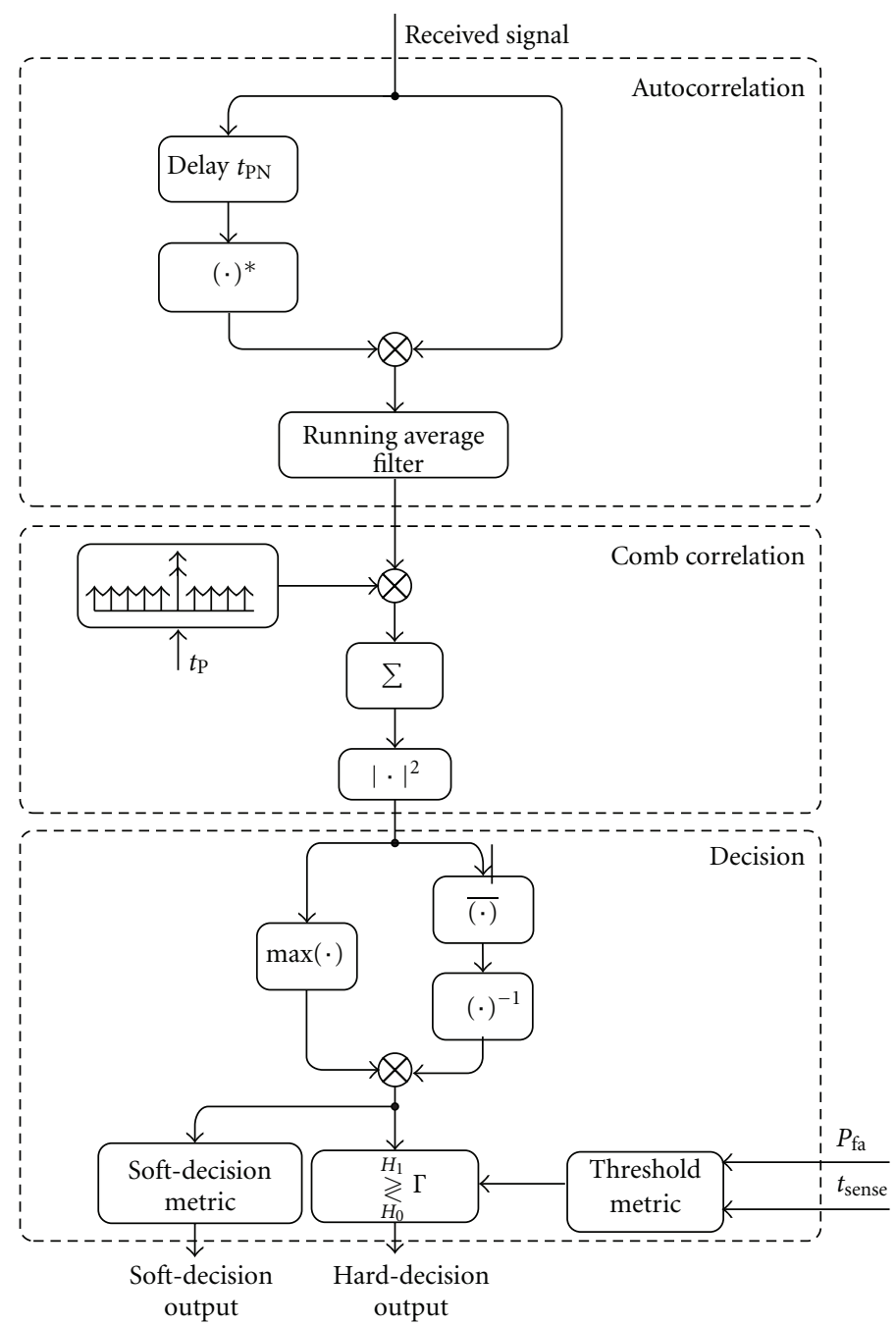

FIgURE 6: Flow diagram of the DTMB sensing algorithm.

$t_{\mathrm{H}}=64 \mu \mathrm{s}$. A flow diagram of the PAL-D/K sensing algorithm is depicted in Figure 7 and consists of two stages:

(i) autocorrelation stage,

(ii) decision stage.

While the delay in the autocorrelation corresponds to the periodicity of the CVBS signal, the length of the running average filter is set to $t_{\mathrm{H}}$ as well. This improves the sensing performance by exploiting similarities in the video signal for consecutive lines. In the decision stage, the average of the output of the autocorrelation stage is calculated. The residual parts of the decision stage are identical to the corresponding parts in the decision stages for DTMB and CMMB signals.

\section{Spectrum Sensing Prototype Platform}

This section presents the underlying prototype platform for the spectrum sensing application. The spectrum sensing prototype presented here is a functional module as part of a TVWS device exploiting the non-occupied frequency for a secondary communication system. The TVWS device can be a base station or user equipment. The sensing prototype must be able to interact with the TVWS device. A synchronization is necessary to match the quiet periods used for the sensing operation. Therefore, two interfaces are defined for the sensing prototype:

(i) Interface to the external clock source by which the prototype can get the synchronization signal for the TVWS device.

(ii) Interface with the spectrum management entity by which the sensing requests and sensing results can be transmitted.

A block diagram of the spectrum sensing entity is shown in Figure 8. It consists of an RF front end, a mixedsignal daughter card, and a C6455 DSP (Digital Signal Processor) Starter Kit (DSK). Core part of the RF front end is a commercially available tuner module. It receives the RF signal by an antenna and down-converts it to an intermediate frequency (IF). This analog IF signal is given to the mixed-signal daughter card and digitized using 


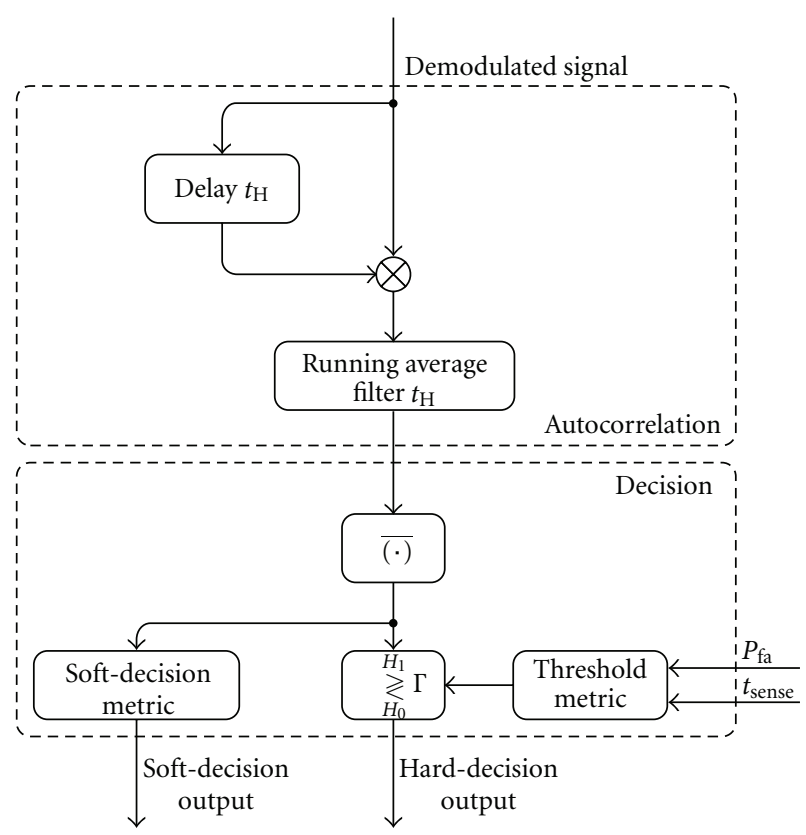

FIGURE 7: Flow diagram of the PAL-D/K sensing algorithm.

an AD6655 analog-to-digital converter (ADC) by Analog Devices. Further filtering and decimation is carried out on the FPGA (Field-Programmable Gate Array) residing on the daughter card as well. The digital baseband signal is transferred to the DSP located on the DSK. The transfer is carried out via DMA (Direct Memory Access) using the Texas Instruments proprietary EMIF (External Memory Interface). The DSP itself is a powerful Texas Instruments TMS320C6455 running at 1.2 GHz system clock. The sensing algorithms for the various TV standards are carried out on the DSP and the results are transferred to the spectrum management entity which is implemented as a program running on a PC. The connection is realized using an Ethernet interface.

The signal flow within the DSP is illustrated in Figure 9. The digital baseband data coming from the FPGA is stored in a local buffer on the DSP. A control unit, which itself is directly controlled by the spectrum management entity, defines to which detectors the signal is passed. There are different operation modes depending on the a priori knowledge about the underlying TV usage. In case the frequency band of interest may only be used by one TV standard, this information is communicated to the control unit so that only the corresponding detection algorithm is carried out. Otherwise, in case this frequency band may be used by any of the available TV standards, the control unit passes the captured data first to the DTMB detector followed by the CMMB detector and, finally, the PAL-D/K detector. The soft-decision outputs of all detectors are then processed by a combination metric to give an overall information about the presence of any of these signals.

A graphical user interface (GUI) has been developed which allows a simple configuration of the sensing parameters and an immediate demonstration of the sensing results.
The synchronization signals are generated by a commercially available Huawei LTE eNodeB. Its signals are received and decoded by the spectrum sensing entity for synchronization with the LTE (Long-Term Evolution) data transmission.

A photography of the spectrum sensing prototype is given in Figure 10. It shows the three aforementioned modules with the RF front end at the top and the DSK at the bottom. In between, the PCB of the daughter card is located. Additionally, a separate PCB is located on the righthand side for debugging purposes and for interfacing with the synchronization entity.

\section{Results}

This section presents both simulation results and laboratory measurement results for the previously presented sensing algorithms. The TV signals are generated by a Rohde \& Schwarz signal generator. The signals are sent to the prototype module for detection. Additionally, the actual signal power is measured using a Rohde \& Schwarz power meter. The parameters used for the simulations as well as for the measurements are as follows: the bandwidth used for all TV standards is $8 \mathrm{MHz}$ and the sensing interval $t_{\text {sense }}$ is set to $20 \mathrm{~ms}$. The target false-alarm probabilities are chosen to be $10 \%$ and $0.1 \%$, while the target detection probability is $90 \%$. For the simulations, a noise figure of $8 \mathrm{~dB}$ is considered. Figure 11 shows the detection probability $P_{\mathrm{d}}$ versus the received signal power $p_{\mathrm{rx}}$. The considered DTMB signal uses frame header mode 1. The blue curves show the simulation results for a false-alarm probability of $10 \%$ and $0.1 \%$, respectively. The red curves show the corresponding measurement results. For the target detection probability of $90 \%$, the measurement results are $3 \mathrm{~dB}$ to $4 \mathrm{~dB}$ worse than the simulation results. Thus, with the given algorithms, a sensitivity of approximately $-110 \mathrm{dBm}$ and $-108.5 \mathrm{dBm}$ can be reached in the presented hardware setup. There are several reasons that could result in such degradations. The simulations assume a perfect AGC while in the real system the maximum gain is limited by the tuner module leading to an increased quantization noise in case of very low signal powers at the input of the tuner. The TV tuner shows highly unstable behavior in terms of amplitude and phase when the input signal is very weak. Such a property results from the fact that the tuner is designed for receiving TV signals at significantly higher power levels. The required sensing sensitivity is much higher than the TV receiver sensitivity. This causes unexpected distortion when the signal level is below the target receiver sensitivity. This aspect is also the reason why autocorrelation algorithms are favorable compared to cross-correlation algorithms. Crosscorrelation algorithms suffer more seriously from such distortions of the tuner, leading maximally to the same overall performance as the autocorrelation algorithms although in simulations such cross-correlation algorithms perform better than their autocorrelation counterparts. However, the computational complexity of cross-correlation implementations is much higher and, hence, autocorrelation algorithms are preferred. Further reasons for the difference between the 


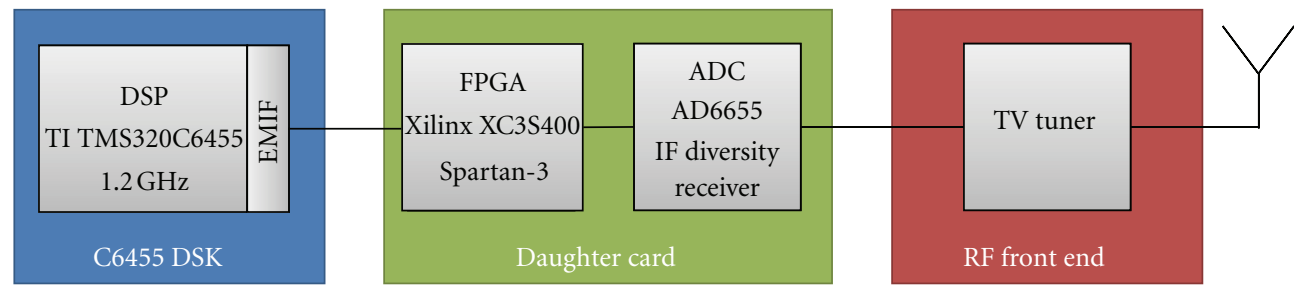

FIGURE 8: Spectrum sensing entity.

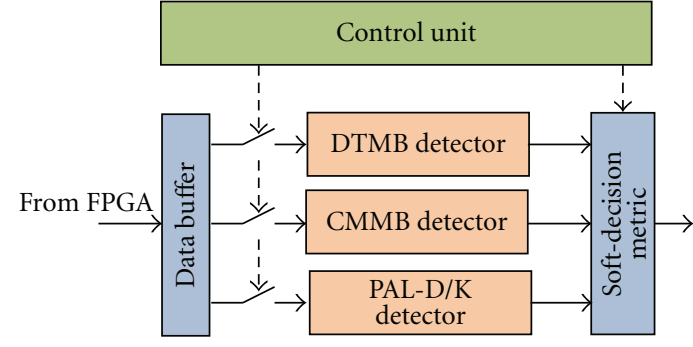

Figure 9: DSP signal flow.

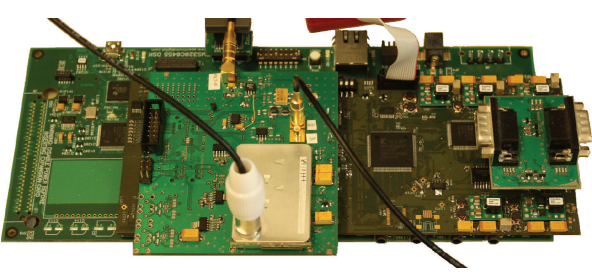

FIGURE 10: Spectrum sensing entity.

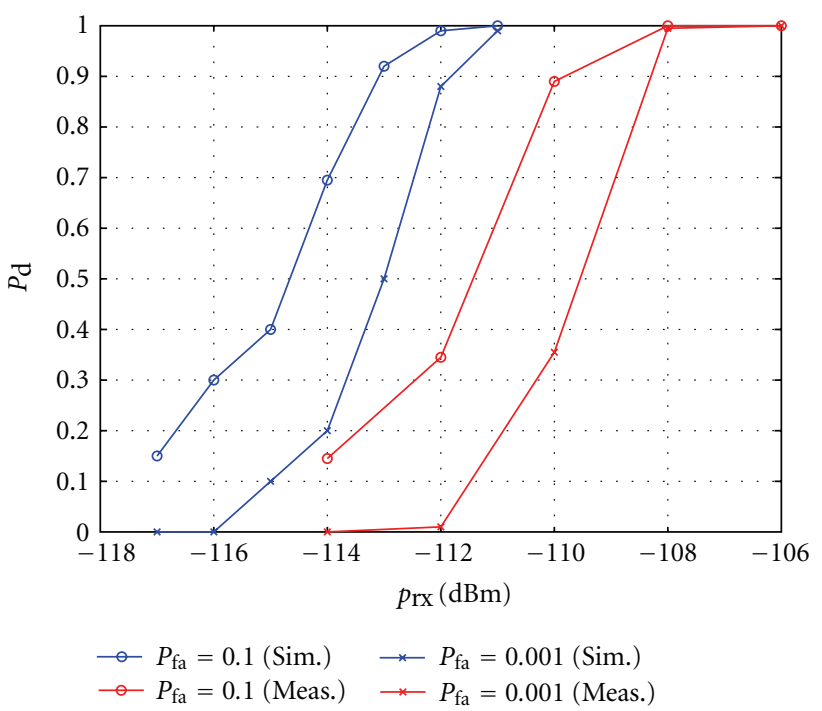

FIGURE 11: Simulation and measurement results for DTMB $\left(t_{\text {sense }}=\right.$ $20 \mathrm{~ms})$.

simulated and measured sensing results are effects such as frequency offsets and amplifier non-linearities in the RF stage which cannot be avoided in hardware implementations and may lead to significant performance degradations.

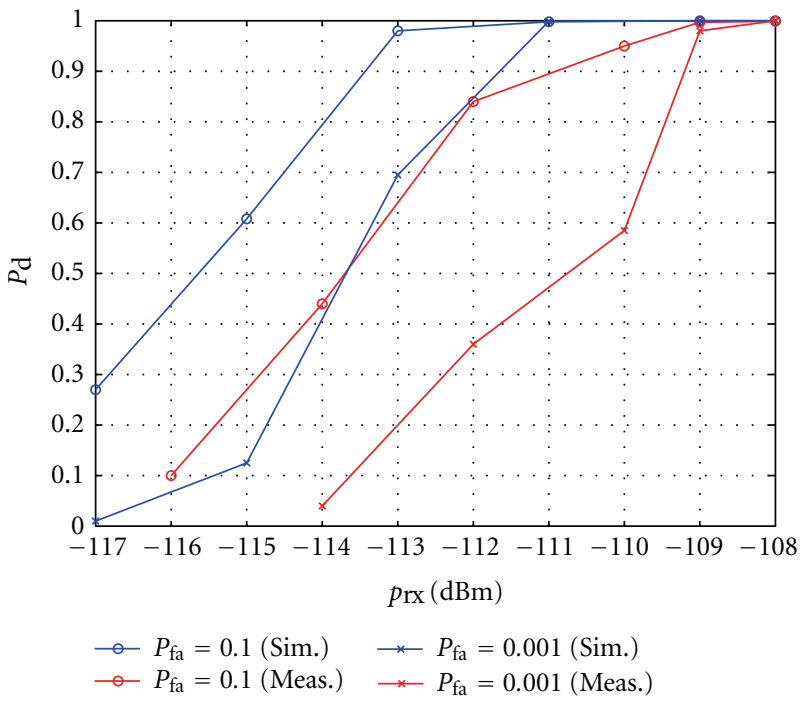

FIGURE 12: Simulation and measurement results for CMMB $\left(t_{\text {sense }}=\right.$ $20 \mathrm{~ms})$.

However, these effects have not been considered in the simulations.

Figure 12 shows the results for CMMB. Again, a degradation of the measurement performance of almost $3 \mathrm{~dB}$ compared to the simulation performance can be identified. With the implemented algorithms, a sensitivity of $-111 \mathrm{dBm}$ $\left(P_{\mathrm{fa}}=10 \%\right)$ and $-109.5 \mathrm{dBm}\left(P_{\mathrm{fa}}=0.1 \%\right)$, respectively, can be reached for the given target detection probability of $90 \%$.

For PAL-D/K, the measured performance is much worse than the simulated performance as shown in Figure 13. One contribution to the performance loss stems from the additional signal processing steps, which are necessary to extract the CVBS signal from the received PAL-D/K signal. These signal processing steps need to be carried out before the sensing operation. However, they are implemented in a way to minimize the processing latency rather than for utilizing the dynamic range most efficiently. This leads to a significant performance degradation in comparison to the simulation results which are based on floatingpoint calculations without any constraints regarding the dynamic range. The sensing threshold for PAL-D/K is about $-102 \mathrm{dBm}$.

The simulation results reveal that the sensing algorithms are suitable to fulfill the Chinese spectrum sensing requirements. However, in case of the digital TV standards DTMB 


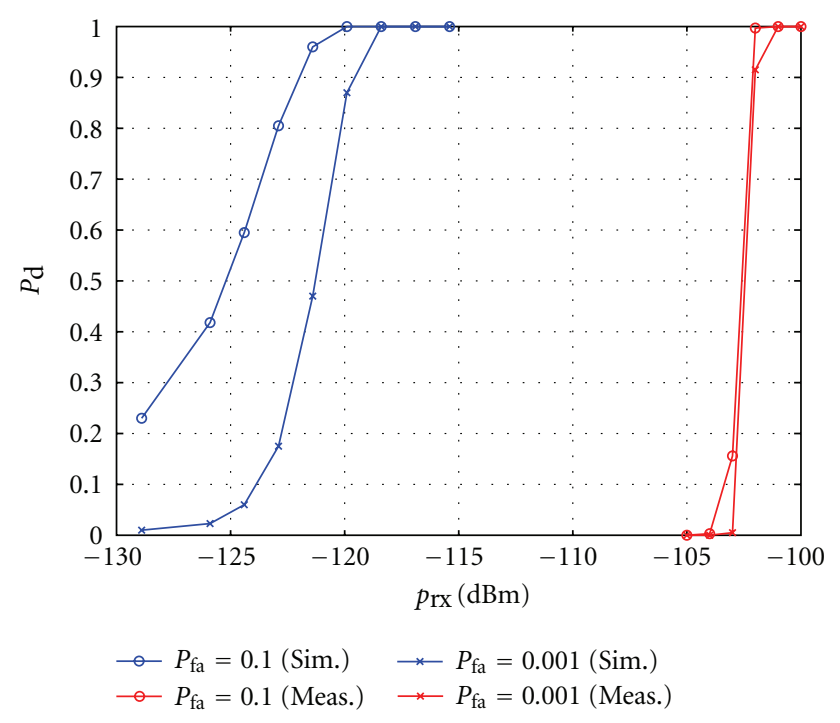

Figure 13: Simulation and measurement results for PAL-D/K $\left(t_{\text {sense }}=20 \mathrm{~ms}\right)$.

and $\mathrm{CMMB}$, the measurements slightly fail the requirements using a sensing interval of $20 \mathrm{~ms}$. In case of the analog TV standard PAL-D/K, the requirements are more relaxed so that even for the significant difference between the simulation results and the measurements, the obtained results are sufficient for the Chinese spectrum sensing requirements.

\section{Conclusion}

Within this paper, an application of cognitive radio for TV white space in China was addressed. Therefore, spectrum sensing algorithms for the three predominant TV standards in China were presented. A prototype platform was introduced and the signal flow was illustrated allowing to sense for the TV standards DTMB, CMMB and PAL$\mathrm{D} / \mathrm{K}$. The performance results revealed that the developed platform allows signal detection even at very low levels of the receive power. Considering a false-alarm probability of $10 \%$ and a target detection probability of $90 \%$, the prototype platform achieves a sensitivity of $-110 \mathrm{dBm}$ for DTMB while for CMMB and PAL-D/K the sensitivity is $-111 \mathrm{dBm}$ and $-102 \mathrm{dBm}$, respectively.

\section{References}

[1] J. Van De Beek, J. Riihijärvi, A. Achtzehn, and P. Mähönen, "UHF white space in Europe-a quantitative study into the potential of the $470-790 \mathrm{MHz}$ band," in Proceedings of the IEEE International Symposium on Dynamic Spectrum Access Networks (DySPAN '11), pp. 1-9, May 2011.

[2] FCC, "Report 10-174: in the matter of unlicensed operation in the TV broadcast bands, additional spectrum for unlicensed devices below $900 \mathrm{MHz}$ and in the $3 \mathrm{GHz}$ bandsecond memorandum opinion and order," Tech. Rep. 10-174, September 2010.

[3] IEEE, "IEEE 802.22 Working Group on Wireless Regional Area Networks," http://www.ieee802.org/22.
[4] W. Fischer, Digital Video and Audio Broadcasting Technology: A Practical Engineering Guide, Springer, Berlin, Germany, 3rd edition, 2010.

[5] Std. GB 20 600-2006, "Framing structure, channel coding and modulation for digital television terrestrial broadcasting system," Std. GB 20 600-2006, August 2006.

[6] Std. GY/T 220.1-2006, "Mobile multimedia broadcasting part 1 -frame structure, channel coding and modulation for broadcasting channel, SARFT - the state administration of radio, film and television," Std. GY/T 220.1-2006, 2006.

[7] Std. GB 3174-1995, "Characteristics of PAL-D television broadcasting system," Std. GB 3174-1995, December 1995.

[8] IEEE Std. 802.22, "Standard for cognitive wireless regional area networks (RAN) for operation in TV band," IEEE Std. 802.22, July 2011.

[9] FCC, Evaluation of the Performance of Prototype TV-Band White Space Devices Phase II, October 2008.

[10] V. Gaddam and M. Ghosh, "Robust sensing of DVB-T signals," in Proceedings of the IEEE Symposium on New Frontiers in Dynamic Spectrum (DySPAN '10), pp. 1-8, April 2010.

[11] A. Xu, Q. Shi, Z. Yang, K. Peng, and J. Song, "Spectrum sensing for DTMB system based on PN cross-correlation," in Proceedings of the IEEE International Conference on Communications (ICC'10), pp. 1-5, May 2010.

[12] L. Wenqi, X. Ning, G. Lijun, Z. Yingxin, and W. Hong, "Spectrum sensing methods for DTMB based cognitive radio systems," in Proceedings of the 1st International Conference on Information Science and Engineering (ICISE '09), pp. 27302733, December 2009.

[13] ETSI Std. EN 300744 V1.6.1, "Digital Video Broadcasting (DVB): framing structure, channel coding and modulation for digital terrestrial television (DVB-T)," ETSI Std. EN 300744 V1.6.1, January 2009. 

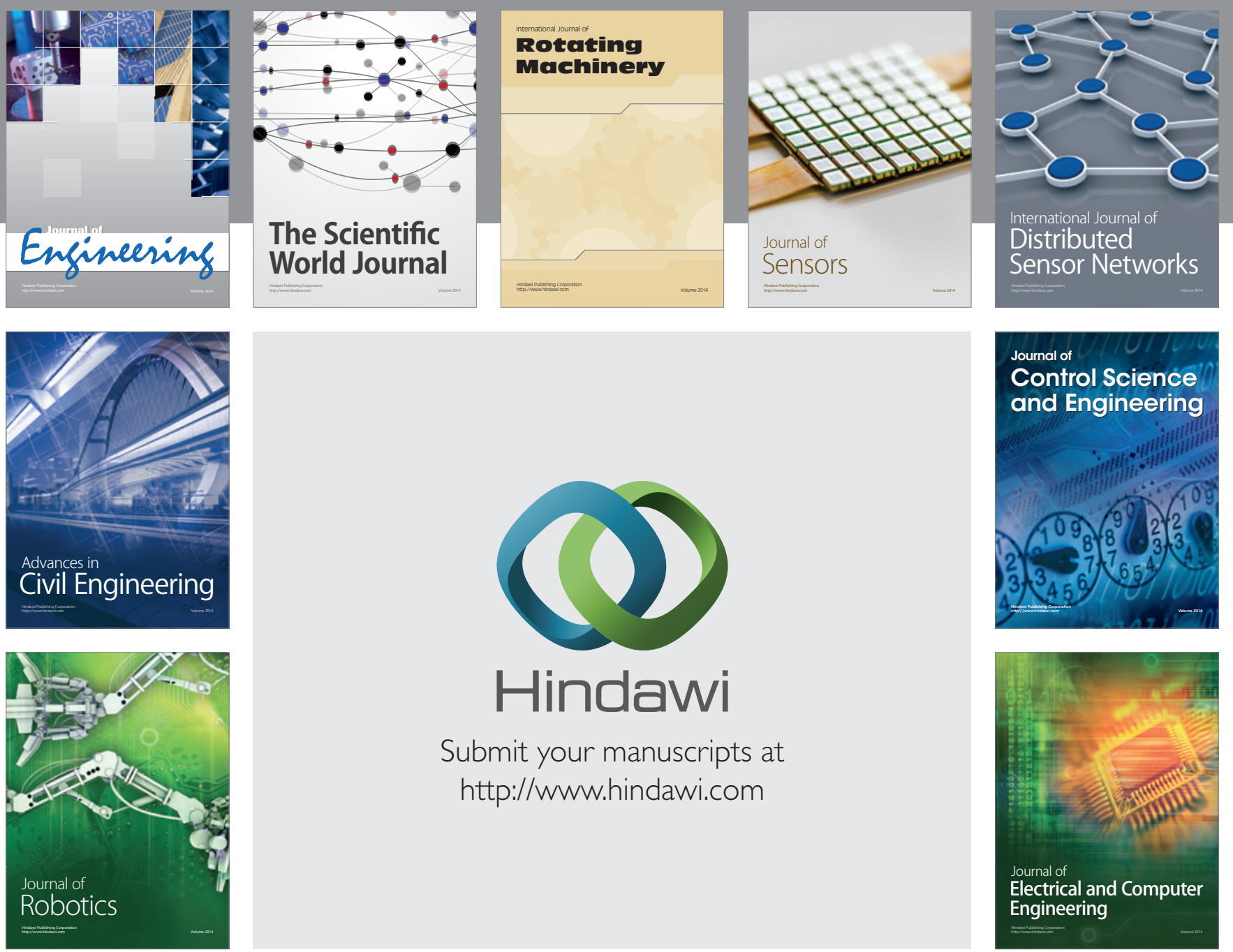

Submit your manuscripts at

http://www.hindawi.com
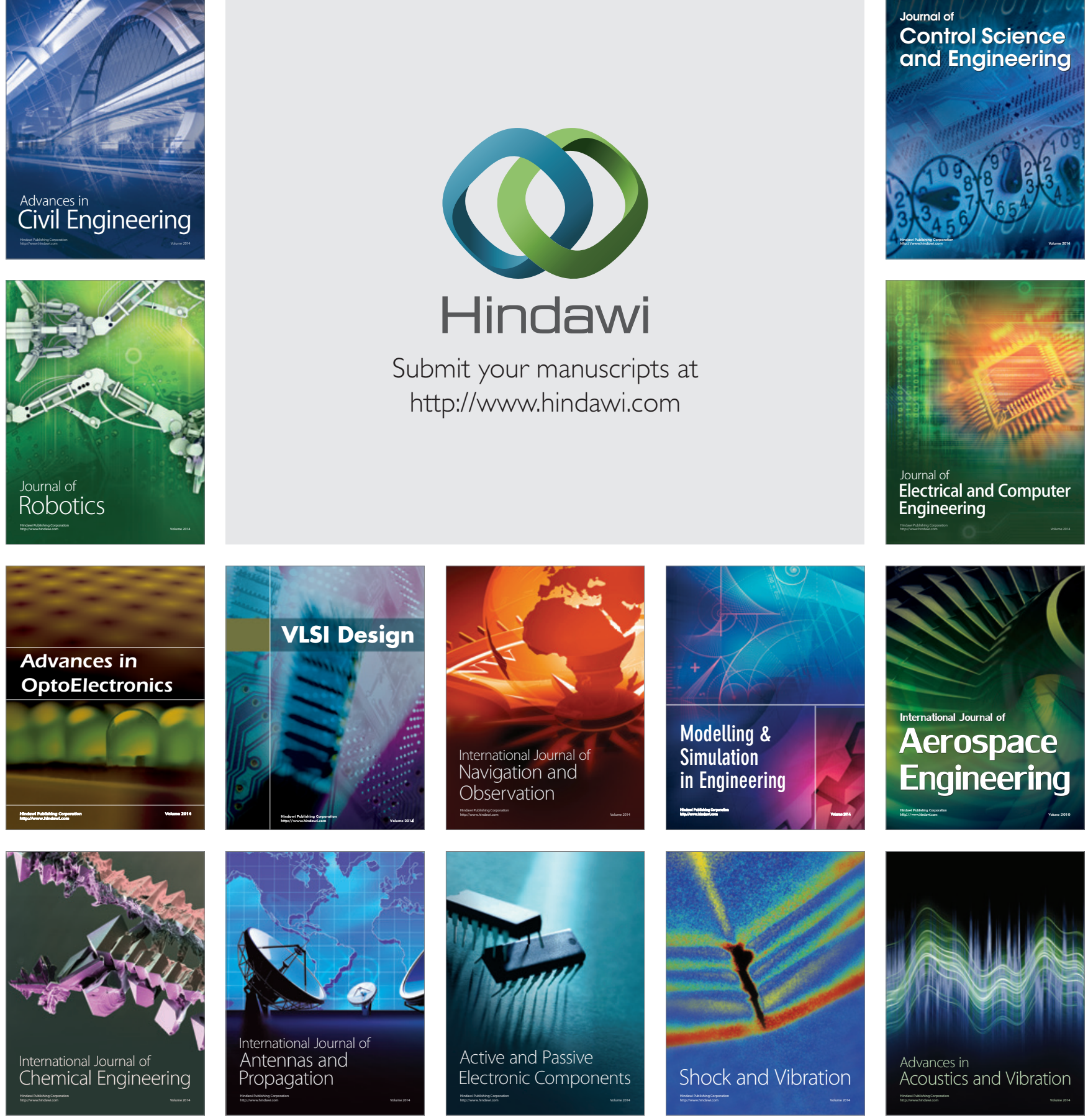